\title{
CONSUMO DE DROGAS EN ESTUDIANTES DE CENTROS ESCOLARES EN SAN SALVADOR, EL SALVADOR
}

\author{
Fabio Bautista Pérez \\ Universidad Internacional Iberoamericana (UNINI) (México) \\ fabioverano120@gmail.com \\ https://orcid.org/0000-0003-3565-7515
}

\begin{abstract}
Resumen. Los estudiantes en el nivel primario de educación por estar en la etapa de la adolescencia están en peligro de ser inducidos al consumo de drogas, por lo que es importante realizar estudios sobre su consumo. Uno de los objetivos del estudio fue analizar las diferencias en el consumo de alcohol, tabaco y marihuana en los adolescentes con relación a la edad y al género. La investigación fue realizada en escuelas de educación básica ubicadas en la ciudad de San Salvador, El Salvador, Centro América. El estudio fue no experimental con un enfoque descriptivo, multivariado y con un diseño trasversal y ex post facto. La población de estudio estuvo conformada por estudiantes de primaria y secundaria de 12 escuelas con una población de 3356 estudiantes, la muestra fue de 345. El inicio de consumo de alcohol, tabaco y marihuana por los estudiantes fue más frecuente a las edades de 12 a 14 años; los varones tuvieron un consumo más alto que las féminas para la prevalencia una vez en la vida del consumo de alcohol, tabaco y marihuana. Para los últimos 12 meses el consumo de tabaco fue ligeramente mayor en el género femenino que el masculino, el consumo de alcohol fue similar en ambos géneros y el consumo de marihuana fueron iguales para ambos. Para los últimos 30 días el consumo de tabaco y alcohol fue similar en ambos géneros y el consumo de marihuana fueron iguales.

Palabras clave. Consumo de drogas, alcohol, tabaco, marihuana, El Salvador.
\end{abstract}

\section{DRUG CONSUMPTION IN STUDENTS OF SCHOOL CENTERS IN SAN SALVADOR, EL SALVADOR}

\begin{abstract}
Students at the primary level of education because they are in the adolescence stage are in danger of being induced to use drugs, so it is important to conduct studies on their consumption. One of the objectives of the study was to analyze the differences in the consumption of alcohol, tobacco and marijuana in adolescents in relation to age and gender. The research was conducted in basic education schools located in the city of San Salvador, El Salvador, Central America. The study was non-experimental with a descriptive, multivariate approach and with a transverse and ex post facto design. The study population consisted of elementary and secondary students from 12 schools with a population of 3356 students, the sample was 345 . The beginning of alcohol, tobacco and marijuana use by students was more frequent at the ages of 12 to 14 years; males had a higher consumption than females for prevalence once in a lifetime of alcohol, tobacco and marijuana use. For the last 12 months, tobacco consumption was slightly higher in women than men, alcohol consumption was similar in both genders and marijuana use was the same for both. For the last 30 days the consumption of tobacco and alcohol was similar in both genders and the consumption of marijuana were the same.
\end{abstract}

Keywords: Drug use, alcohol, tobacco, marijuana, El Salvador. 


\title{
CONSUMO DE DROGAS EM ALUNOS DE CENTROS ESCOLARES EM SAN SALVADOR, EL SALVADOR
}

\begin{abstract}
Resumo. Os alunos do ensino fundamental, por estarem na adolescência, correm o risco de serem induzidos a usar drogas, por isso é importante realizar estudos sobre o consumo. Um dos objetivos do estudo foi analisar as diferenças no consumo de álcool, tabaco e maconha em adolescentes em relação à idade e sexo. A pesquisa foi realizada em escolas de educação básica localizadas na cidade de San Salvador, El Salvador, América Central. O estudo não foi experimental, com abordagem descritiva, multivariada e com delineamento transversal e ex post facto. A população do estudo foi composta por alunos do ensino fundamental e médio de 12 escolas, com uma população de 3.356 alunos, a amostra foi de 345 . O início do uso de álcool, tabaco e maconha pelos estudantes foi mais frequente entre os 12 e os 12 anos. 14 anos; os homens tiveram um consumo maior do que as mulheres em prevalência uma vez na vida em uso de álcool, tabaco e maconha. Nos últimos 12 meses, o uso de tabaco foi levemente maior em mulheres que em homens, o consumo de álcool foi semelhante em ambos os sexos e o uso de maconha foi o mesmo em ambos. Nos últimos 30 dias, o consumo de tabaco e álcool foi semelhante em ambos os sexos e o consumo de maconha foi o mesmo.
\end{abstract}

Palavras chave. Uso de drogas, álcool, tabaco, maconha, El Salvador.

\section{Introducción}

De acuerdo con la organización mundial de la Salud (OMS, 2015), se estima que un total de 246 millones de personas, o una de cada 20 personas de edades comprendidas entre los 15 y 64 años, consumieron drogas ilícitas en 2013. Si bien ello representa un aumento de tres millones de personas con respecto al año anterior, debido al crecimiento de la población mundial, en realidad el consumo de drogas ilícitas se ha mantenido estable, así también hace mención que las drogas causan alrededor de medio millón de muertos anuales y que, en algunos aspectos, la situación ha empeorado en los últimos años. Esta cifra contrasta con la ofrecida por la Oficina de Naciones Unidas contra la Droga y el Delito (ONUDD), que el año pasado estimó que las muertes debido al consumo de drogas eran de poco más de 200.000 personas. "En algunos aspectos, la situación está empeorando y no mejorando. Muchos países están experimentando una crisis de emergencia sanitaria debido a las muertes por sobredosis", agregó la directora de la OMS. Un informe de la Junta Internacional de Fiscalización de Estupefacientes (JIFE) ha indicado que en EE. UU. casi se duplicaron los fallecimientos por sobredosis entre 2013 y 2014, cuando en este último año se registraron más de 47.000 muertes por esa causa. Con relación a esto la directora de la OMS pidió a los 53 países de la Comisión que se aborde el consumo de drogas como un problema de salud pública y no con medidas penales. Entre los países de la Comisión se encuentran Irán y China, países con castigos severos para el consumo de drogas y el narcotráfico, que pueden incluso llegar a la pena de muerte (OMS, 2016, 2017).

El fenómeno de las drogas es un problema de dimensiones mundiales, el cual se ha agudizado por la globalización, ya que a través de ella hay una progresiva difusión de hábito e ideas a través de redes y medios de comunicación masiva que incitan al consumo y tráfico de drogas. La magnitud del problema mundial de las drogas se hace más evidente porque más de 1 de cada 10 consumidores de drogas es un consumidor problemático que sufre trastornos ocasionados por el consumo de drogas o drogodependencias, lo que supone una pesada carga para los sistemas de salud de los países (OMS, 2015, Ortiz Rebolledo y Silva Costa, 2005). 
De acuerdo con Ortiz Rebolledo y Silva Costa (2005), el abuso de drogas abarca todos los ámbitos de la convivencia social, ya que su relación con las enfermedades, la violencia, la delincuencia y la pobreza es ampliamente reconocida. En este sentido la comunidad internacional desarrolla importantes esfuerzos por controlar su producción, comercialización y consumo, además de intentar contener las graves repercusiones que tiene en la salud y su consiguiente costo social.

La república de El Salvador, a través de instituciones como la Comisión Nacional Antidrogas (CNA), tiene como objetivo establecer un sistema nacional de indicadores con información estadística sobre drogas confiable y actualizada para orientar la toma de decisiones de los funcionarios, en la definición y evaluación de políticas, programas y proyectos relacionados con la reducción de la demanda y reducción de la oferta de drogas de acuerdo al Ministerio de Justicia y Seguridad Pública (MJSP, 2009).

A partir del año 2001, El Salvador forma parte del Sistema Interamericano de datos Uniformes sobre Consumo de Drogas (SIDUC). En el marco del Primer Plan Nacional antidrogas 2002-2008, el país estableció como parte de las metas a ejecutar la realización de encuestas de prevalencia de consumo de drogas en estudiantes de centros escolares a nivel nacional (MJSP, 2009).

De esta manera el año 2003, con el apoyo técnico y financiero del Observatorio Interamericano sobre Drogas de la CICAD-OEA, se realizó la "Primera Encuesta de Prevalencia de Consumo de Drogas en Jóvenes de Centros Escolares Urbanos Mayores de 30,000 estudiantes", con el fin de caracterizar los patrones de consumo de drogas en la población escolar a nivel nacional (MJSP, 2009).

Con relación a la prevención de consumo de drogas el Ministerio de Educación (MINED) ha implementado el Programa Educación para la Vida, que es un complemento al programa formal que desarrollan los docentes en todas las escuelas con estudiantes de 10 a 15 años, donde se ha aplicado al currículo nacional componentes de educación en derechos humanos-prevención del consumo indebido de drogas y otros componentes, los cuales se trabajan en las aulas docente/estudiantes, entre las escuelas y escuela de padres y madres (MINED, 2008).

En España, Martínez Higueras (2012), en su trabajo de Tesis Doctoral encontró que en adolescentes (14-18 años), casi el 50\% habían consumido alcohol, seguido por el tabaco con casi $40 \%$ y cannabis el $16 \%$. Otro estudio de carácter nacional mostró en estudiantes de 14 a 18 años un consumo más elevado de alcohol (81.9\%), tabaco (35.3\%) y marihuana (26.6\%) de acuerdo con Ministerio de Sanidad, Servicios Sociales e Igualdad (ESTUDES, 2013).

En un estudio nacional sobre consumo de drogas en población escolar de la república de Chile según el Servicio Nacional para la Prevención y Rehabilitación del Consumo de Drogas y Alcohol (SENDA, 2014), se observó una disminución del uso diario de tabaco entre escolares de $42 \%$ a $26.7 \%$ con relación a 2011 . Con relación al alcohol en el último mes alcanzó el $35.6 \%$, esto es una variación de 0.9 puntos porcentuales respecto al 2011, habiendo un incremento tanto en hombres como en mujeres. Para el caso de la marihuana en el último año ha aumentado significativamente, llegando a un $30.6 \%$, lo que equivale a $11.1 \%$ más que los registrados el estudio anterior. El consumo de marihuana se mantuvo estable en $3.6 \%$ con un leve incremento de $0.4 \%$ con relación al estudio anterior. La pasta base se consumió en $2.3 \%$ permaneciendo estable. El consumo de inhalantes se incrementó a 5.2\% en el último año. Los tranquilizantes sin receta médica se incrementó su consumo a $9.5 \%$ en el último año, siendo mayor en mujeres que en hombres. 
En El Salvador, las mayores prevalencias de consumo de sustancias psicoactivas se concentran en el consumo de sustancias legales como alcohol y tabaco, tanto en las prevalencias de vida, año y mes, lo cual ha sido informado a través de diversos estudios realizados en diferentes años por la Comisión Nacional Antidrogas (CNA, 2014a y CNA, 2014b)

El consumo de drogas lícitas e ilícitas en El Salvador se ha incrementado de acuerdo con estudios realizados por la (CNA, 2008) y otros estudios realizados por universidades y ONG's de acuerdo con la CNA. Esto hace necesario que se investigue porqué las prácticas de prevención del consumo de drogas en estudiantes de educación básica no ha reducido los índices de consumo.

El consumo de sustancias legales e ilegales constituye uno de los problemas más importantes con los que se enfrenta la sociedad actual, ya que ocasiona graves costos personales, sociales y económicos. Además, esta conducta es más preocupante cuando se produce en población infantil y juvenil, no sólo por los problemas inmediatos que puede generar, sino también por la consolidación del consumo que implica su inicio en edades tempranas. El aumento en el índice de consumo de drogas entre adolescentes es cada vez mayor y el problema parece cada día más difícil de resolverse, por lo que nuevos programas de prevención de drogas son importantes para disminuir el consumo en adolescentes (Martínez-Lorca y Alonso-Sans, 2003). Entre los principales problemas o conflictos que el consumo de drogas produce en los estudiantes cabe destacar el bajo rendimiento escolar, los episodios de agresividad, los accidentes de tráfico y, en general, problemas relacionados con la convivencia social. Además, el consumo de sustancias lícitas e ilícitas depende de factores personales, psicológicos, sociales y económicos que interactúan con las características farmacológicas de cada droga, de acuerdo con varios estudios realizados (CNA, 2005).

En los países desarrollados entre un tercio y la mitad de los escolares experimentan con el tabaco antes de finalizar la escolarización secundaria. En Estados Unidos de acuerdo con la encuesta escolar sobre tabaco, alcohol y cannabis Monitoring the Future (MTF), mostró que a los 15 años el $26 \%$ de los adolescentes fumaron al menos un cigarrillo en los últimos 30 días de acuerdo con Bachman, Johnston, O'Malley y Humphrey (1988).

El consumo de alcohol, tabaco y otras drogas producen cada año más muertes, enfermedades y discapacidades que cualquier otra causa susceptible de ser prevenida. El costo económico que supone esta epidemia para todas las administraciones públicas es enorme, pero más importante aún es la mortalidad que conlleva, esto de acuerdo con los autores citados por Becoña, (2002a). Dado que las técnicas de tratamiento no consiguen la eficacia que a todos nos gustaría, una opción más prometedora y con una mejor relación costo/beneficio es el desarrollo y la aplicación de programas de prevención dirigidos a niños/as y adolescentes. La prevención es indispensable para evitar que el consumo de drogas se convierta en un problema social de gran magnitud. Pero ¿qué es la prevención? Para Martín, citado por Becoña (2002b), la prevención de drogodependencias "es un proceso activo de implementación de iniciativas tendientes a modificar y mejorar la formación integral y la calidad de vida de los individuos, fomentando el autocontrol individual y la resistencia colectiva ante la oferta de drogas".

En El Salvador, el consumo de drogas ha sido un fenómeno cada vez más alarmante, en las últimas dos décadas ha afectado cada vez más a los niños y adolescentes, llegando a la distribución y consumo de drogas en centros de educación básica. De acuerdo con la demanda de atención a este fenómeno, en el país se han ido institucionalizando 
mecanismos como el Plan Nacional Antidrogas 2002-2008. En el mencionado plan, se refleja el incremento de la demanda a partir de 1998 que se ha experimentado un aumento en el consumo de crack, cocaína y drogas sintéticas como éxtasis (República de El Salvador, 2011).

De acuerdo con estudios realizados en El Salvador la tendencia del consumo de drogas tiende a incrementarse entre los jóvenes en los diferentes niveles de estudio en que se encuentran, ya sea en la educación básica, secundaria y universitaria. Así se tiene que en estudios realizados en El Salvador por la (CNA, 2008) sobre la prevalencia del consumo de drogas en estudiantes escolares hasta el bachillerato han demostrado que han consumido drogas lícitas e ilícitas. Las drogas lícitas más consumidas fueron el alcohol en porcentajes que oscilaron de $30 \%$ a $45.8 \%$ y el tabaco en porcentajes de 33 a $37.7 \%$; entre las ilícitas se encuentran la marihuana con un consumo de 4.5-6.1\%, inhalantes de 1.5 a $2.8 \%$, cocaína 1.6 a $1.9 \%$ y crack de 1.1 a $1.2 \%$.

De acuerdo al "Segundo estudio nacional sobre consumo de sustancias psicoactivas en población escolar de El Salvador SIDUC 2008”, en estudiantes cursando séptimo grado, noveno grado y segundo año de bachillerato, se encontró que a este nivel de estudio la prevalencia de consumo de substancias como alcohol fue de $32.5 \%$, cigarrillos $27.9 \%$, marihuana $5.5 \%$, tranquilizantes sin prescripción médica $2.8 \%$, solventes e inhalantes $2.8 \%$, clorhidrato de cocaína $1.9 \%$, crack $1.2 \%$, estimulantes sin receta médica $1.7 \%$ y otras sustancias ilícitas como alucinógenos, heroína, crack y éxtasis en menores proporciones (Comisión Nacional Antidrogas, 2006). Parte de esta población estudiantil es la que ingresará a la universidad y de alguna forma tratará de continuar con la costumbre de consumo de sustancias psicoactivas.

El consumir drogas psicoactivas lícitas e ilícitas podrían incidir en el futuro rendimiento del estudiante universitario por lo que conocer la prevalencia de consumo de sustancias psicoactivas en los estudiantes que ingresan a la universidad es de mucha importancia porque a partir de ello la universidad puede establecer políticas de prevención que ayuden a minimizar o erradicar el consumo de drogas.

El consumo de droga es una enfermedad adictiva crónica, una "pandemia" que afecta a todos los grupos étnicos y no tiene fronteras ni limitaciones de género, edad, estrato sociocultural o religión. En la actualidad, el consumo de tabaco es uno de los principales problemas de salud pública en los países desarrollados y comienza a serlo en los países en vías de desarrollo, no sólo por su magnitud en términos del número de sujetos afectados, sino por las enormes consecuencias sociales, económicas y sanitarias que conlleva (Ponciano-Rodríguez, 2007).

Estudios realizados en El Salvador, se encontró que el consumo de drogas son las sustancias más consumidas y que los hombres consumen más que las mujeres. La cifra de personas que mueren a consecuencia del consumo de tabaco en El Salvador solo se compara al número de muertos que causa la violencia diariamente y son más que las provocadas por el VIH, ya que se contabilizan 12 muertes y constituyen la segunda causa de muerte después de la hipertensión arterial en el país (Luis Antonio Torres. Comisión Nacional Antidrogas, CNA. Información sobre consumo de tabaco proporcionada al periódico El Universitario, Universidad de El Salvador, 2011).

Para conocer los patrones de consumo se han realizado estudios variados en los países de la región y en diferentes edades de la población. La Comisión Salvadoreña Antidrogas (COSA, 2000) realizó un estudio con una muestra de 1,615 escolares de 
colegios públicos y privados, y encontró que el alcohol fue la droga que tuvo la mayor oportunidad de uso por los escolares, seguido por el tabaco y la marihuana.

La prevención escolar del consumo de drogas en una muestra de 6,265 estudiantes de secundaria (10 a 15 años) en El Salvador, no ha sido satisfactoria, porque de acuerdo con varios estudios realizados en la Comisión Nacional Antidrogas (CNA, 2008), la tendencia ha sido al incremento. Esto a pesar de que el Ministerio de Educación (MINED) a través del Programa Educación para la Vida, que es un complemento al programa formal que desarrollan los docentes en todas las escuelas con estudiantes de 10 a 15 años, se ha aplicado al currículo nacional componentes de educación en derechos humanos-prevención del consumo indebido de drogas y otros, los cuales se trabajan en las aulas docente/estudiantes, entre las escuelas y escuela de padres y madres (MINED, 2008).

Según estudio realizado por Rivera de Parada (2009), el consumo de drogas es un problema muy antiguo, pero se ha venido proliferando, en la medida que los intereses económicos están por encima de todo valor humano, se ha asociado a las modas juveniles, modelaje de familiares, artistas y personajes famosos promovidos por la publicidad, condición que se fortalece con los problemas sociales como la desintegración familiar, emigración y desempleo, generando frustración, baja auto estima y otras limitaciones personales que predisponen al consumo de drogas en niños, jóvenes y adultos.

Uno de los objetivos propuestos en el estudio fue: Analizar las diferencias en el consumo de alcohol, tabaco y marihuana en los adolescentes con relación a la edad y al género.

\section{Método}

La investigación fue realizada en escuelas de educación básica y secundaria ubicadas en la ciudad de San Salvador, El Salvador, Centro América. La selección de los centros de estudios se hizo en conjunto con las autoridades del Ministerio de Educación de El Salvador (MINED), a quienes se les solicitó su autorización para la realización del estudio. Así mismo, los participantes, se tomaron al azar siguiendo el método de muestreo sistemático mencionado por Hernández, Fernández y Baptista (2014) y Polit y Hungler (2000).

La aplicación del instrumento se realizó en las aulas de clase de las instituciones de educación primaria de séptimo a noveno grado. El cuestionario se proporcionó en formato impreso en papel, siendo aplicado por el investigador y personal asistente previamente entrenado. Durante el llenado se estuvo pendiente de cualquier pregunta o duda que tuvieran los participantes atendiéndoseles con esmero para subsanar sus preguntas o dudas presentadas. El tiempo proporcionado para el llenado fue de una hora y cuando el estudiante finalizaba de llenarlo nos llamaba para entregarlo. Antes de darlo por recibido se revisaba para determinar si se había llenado correctamente; si no estaba correctamente llenado se indicaba al participante para que lo completara o corrigiera lo que estaba incorrecto. Una vez realizadas las correcciones se daba por recibido. Una vez entregado se colocaban en paquetes de bolsas plásticas y se colocaban en cajas de cartón debidamente rotuladas con el nombre del Centro Educativo de donde procedían. Luego se trasladaban a la institución para ser procesadas.

El estudio fue no experimental con un enfoque descriptivo, multivariado y con un diseño trasversal y ex post facto. La información fue tomada del ambiente natural donde se encuentran los estudiantes sin ninguna modificación, ya que de acuerdo con Martínez 
Higueras (2012), "en la práctica real de prevención escolar sobre drogas no es factible ni ético manipular las situaciones de prevención para ver cómo afecta a los consumos y los factores facilitadores de carácter psico-social".

La población de estudio estuvo conformada por estudiantes de primaria y secundaria de 12 escuelas de la ciudad de San Salvador, la población estimada de estas escuelas fue de 3356, siendo la muestra calculada de 345. La muestra fue calculada utilizando el programa libre de internet Survey System Calculator. Este programa está basado en los intervalos de confianza al 95\% de significancia (The Survey System, 2013). La muestra fue ajustada y estratificada posteriormente de acuerdo con imprevistos que se presentaron durante el desarrollo de la investigación siendo la muestra de trabajo de 272 estudiantes.

La opción metodológica escogida para la recogida de datos fue el cuestionario, como una técnica cuantitativa que es una de las utilizadas en este tipo de estudio. Quintero Ordóñez (2015), menciona que el cuestionario posibilita recabar información sobre lo que hacen, opinan o piensan los encuestados, mediante preguntas realizadas por escrito y que pueden ser respondidas en presencia o ausencia del encuestador.

El cuestionario contenía 208 preguntas, las que estaban distribuidas en información general, situación familiar, relación con la familia, situación escolar estudiantil, salud del estudiante, consumo de tabaco, consumo de alcohol, consumo de marihuana, lugares de consumo y otras situaciones, exposición a programas de prevención de consumo de drogas, satisfacción con el programa Educación para la vida para la prevención de drogas y sobre actitudes hacia las drogas.

La estructura del instrumento contenía ítems sobre la empatía, autocontrol, autoestima, asertividad, habilidades sociales, toma de decisiones, afrontamiento y solución de problemas, actitud desfavorable hacia las drogas, actitud sobre la salud, información sobre drogas, problemas o ajuste escolar, actividades de ocio, comunicación con los padres de familia, variables de control como el tiempo de convivencia en el lugar, en el centro escolar, repeticiones de grado, experiencia previa en prevención del consumo de drogas, consumo de alcohol, tabaco y marihuana. De acuerdo con Martínez Higueras (2012, pág. 149) "los ítems se derivan de escalas que valoran los factores de riesgo y protección para el consumo de drogas". El llenado del cuestionario le llevó al estudiante aproximadamente una hora.

La base de datos y el análisis estadístico de la información se realizó con el Programa libre de internet Perfect Statistical Professionally Presented (PSPP), que es una alternativa libre para el análisis de datos (Salmerón Gómez, 2015). Inicialmente se determinó el coeficiente de fiabilidad a través del coeficiente de Alfa de Crombach; luego la información obtenida fue analizada descriptivamente, determinando frecuencias, intervalos de confianza, relaciones de género y grupos de edades con respecto a las variables en estudio. A estas relaciones se les aplicó la prueba de independencia Chi Cuadrado.

En todos los análisis se consideró la existencia de diferencias estadísticas significativas para el valor de $\mathrm{p}<0.05$ (Hernández, Fernández y Baptista, 2014 y Polit y Hungler, 2000). 


\section{Resultados}

La distribución de la muestra por sexo fue de un 51.5\% para el género masculino y $46.7 \%$ del femenino como se puede apreciar en la Tabla 1.

\section{Tabla 1}

Distribución de la muestra por sexo

\begin{tabular}{lcc}
\hline Sexo & Frecuencia & \% \\
\hline Masculino & 140 & $51.5 \%$ \\
Femenino & 127 & $46.7 \%$ \\
No contestaron & 5 & $1.8 \%$ \\
Total & 272 & $100.0 \%$ \\
\hline Nota $:$ Fuente: Elaboración propia &
\end{tabular}

El promedio de edad de la muestra fue de 14.65 años, una Desviación Estándar (DE) 1.24 años, una edad mínima de 12 años y una máxima de 14.65 años (Tabla 2).

Tabla 2

Promedio de edad de la muestra

\begin{tabular}{llrlc}
\hline & Mínima & Máxima & Promedio & Desviación estándar \\
\hline Edad en años & 12 & 19 & 14.65 & 1.24 \\
\hline Nota $:$ Fuente: Elaboración propia & & &
\end{tabular}

Nota: Fuente: Elaboración propia

El consumo de tabaco en los estudiantes de este nivel puede considerarse alto ya que para una vez en la vida el porcentaje fue de $63.2 \%$, para los últimos 12 meses de $32.0 \%$ y para los últimos 30 días existe casi un $6.0 \%$ de consumo, lo cual puede predisponer a los demás compañeros a imitarlos (Figura 1).

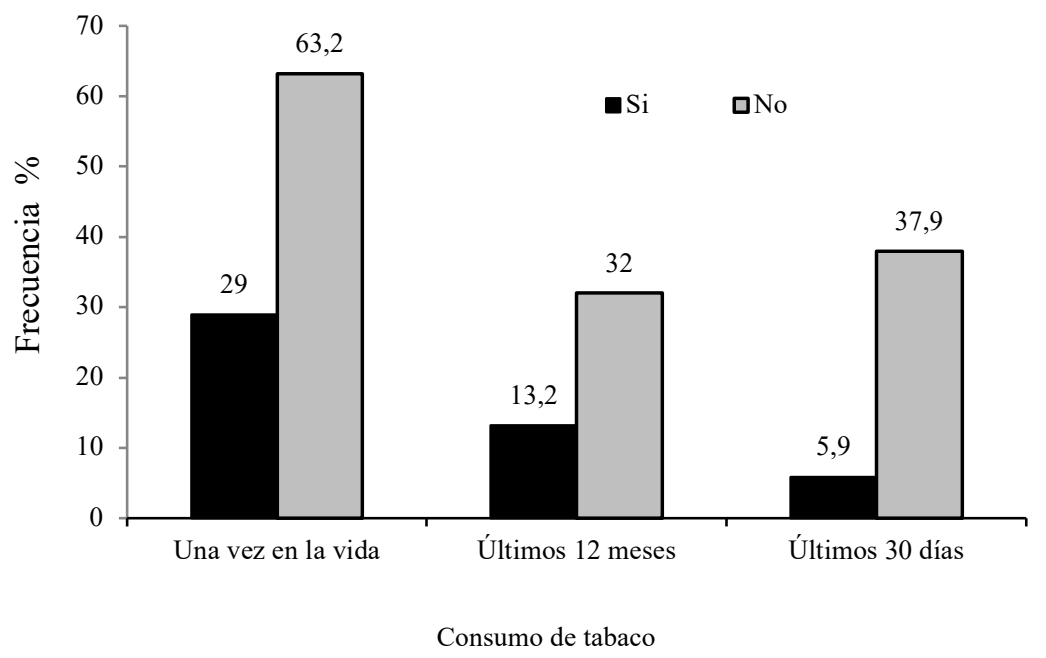

Figura 1. Prevalencia del consumo de tabaco

Fuente: Elaboración propia 
El sexo masculino tuvo un consumo más alto que el femenino para la prevalencia una vez en la vida; en cambio para los últimos 12 meses el consumo fue ligeramente mayor en el género femenino que el masculino y para los últimos 30 días tuvieron un consumo similar (Figura 2). En los tres casos no se encontraron diferencias estadísticas significativas.

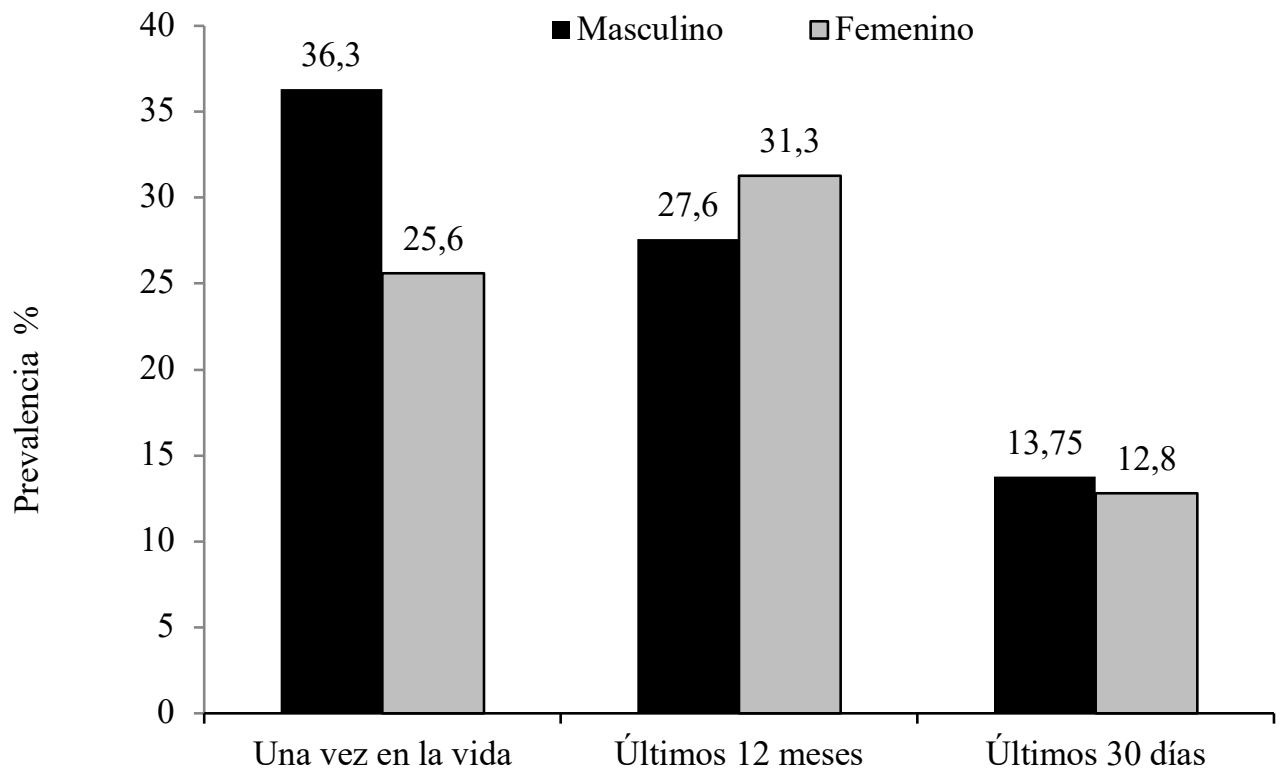

Figura 2. Prevalencia de consumo de tabaco de acuerdo con sexo

Fuente: Elaboración propia

De 78 estudiantes que contestaron a que edad fumaron por primera vez tabaco u otra forma el 7.4\% mencionó que lo hizo a los 13 años, el 7.0\% lo hizo a los 12 años, el $4.8 \%$ lo hizo a los 14 años, 2.6\% lo hizo a los 15 años. También se encontró niños que habían fumado ya a los 5, 9, 10 y 11 años, aunque en porcentajes bajos (Figura 3). 


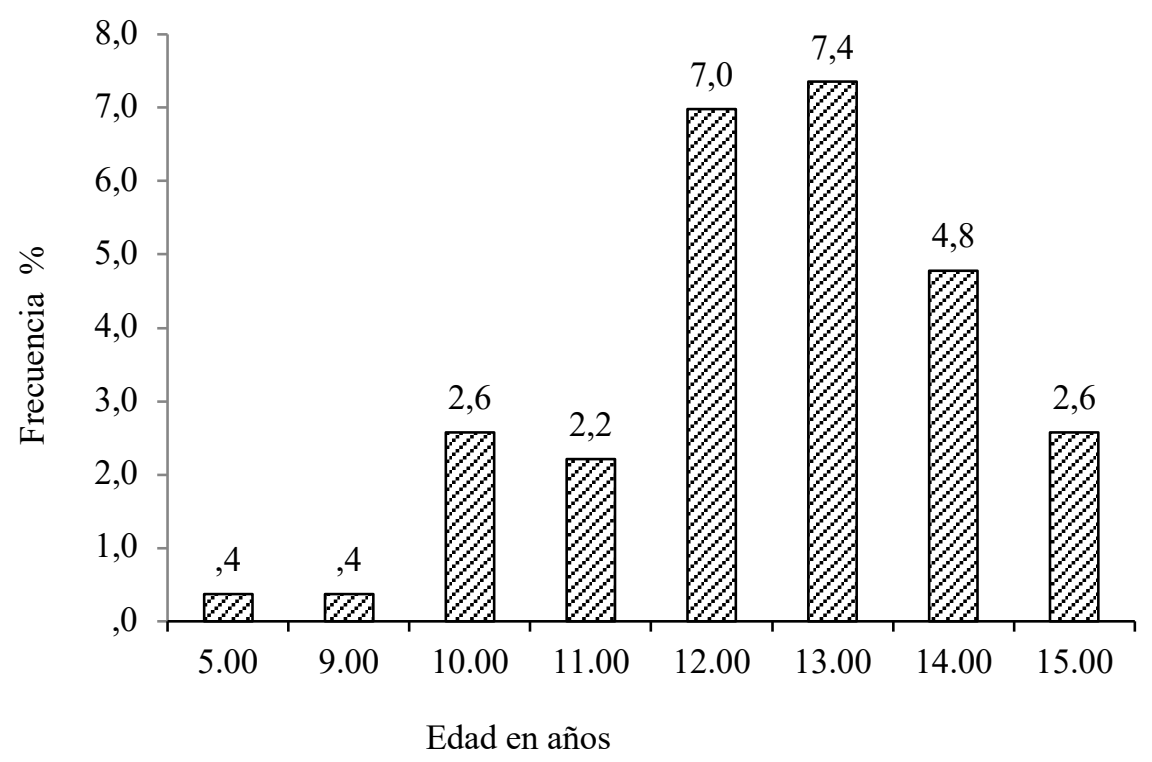

Figura 3. Inicio del consumo de tabaco. $\mathrm{n}=78$

Fuente: Elaboración propia

En general el consumo de alcohol se puede considerar alto, ya que son estudiantes de primaria en edad de la adolescencia, donde no debería de haber ningún consumo, pero como se puede observar en la Figura 4, uno de cada diez estudiantes había consumido alcohol en los últimos 30 días.

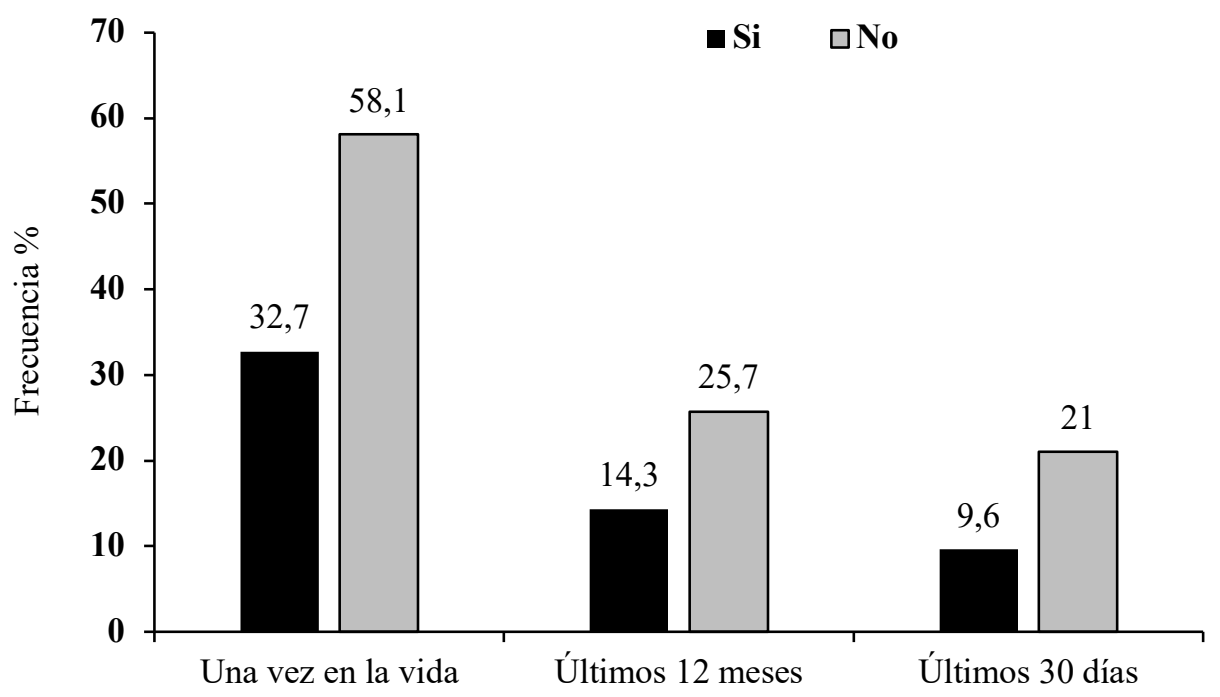

Consumo de alcohol

Figura 4. Prevalencia del consumo de alcohol

Fuente: Elaboración propia 
En relación con el consumo de alcohol entre el sexo masculino y femenino se encontró que el masculino presentó un consumo más elevado que el femenino; así, para una vez en la vida fue de $6.7 \%$ mayor que el femenino, para los últimos 12 meses fue de $2 \%$ y para los últimos 30 días fue del 3\%, como puede observarse en la Figura 5.

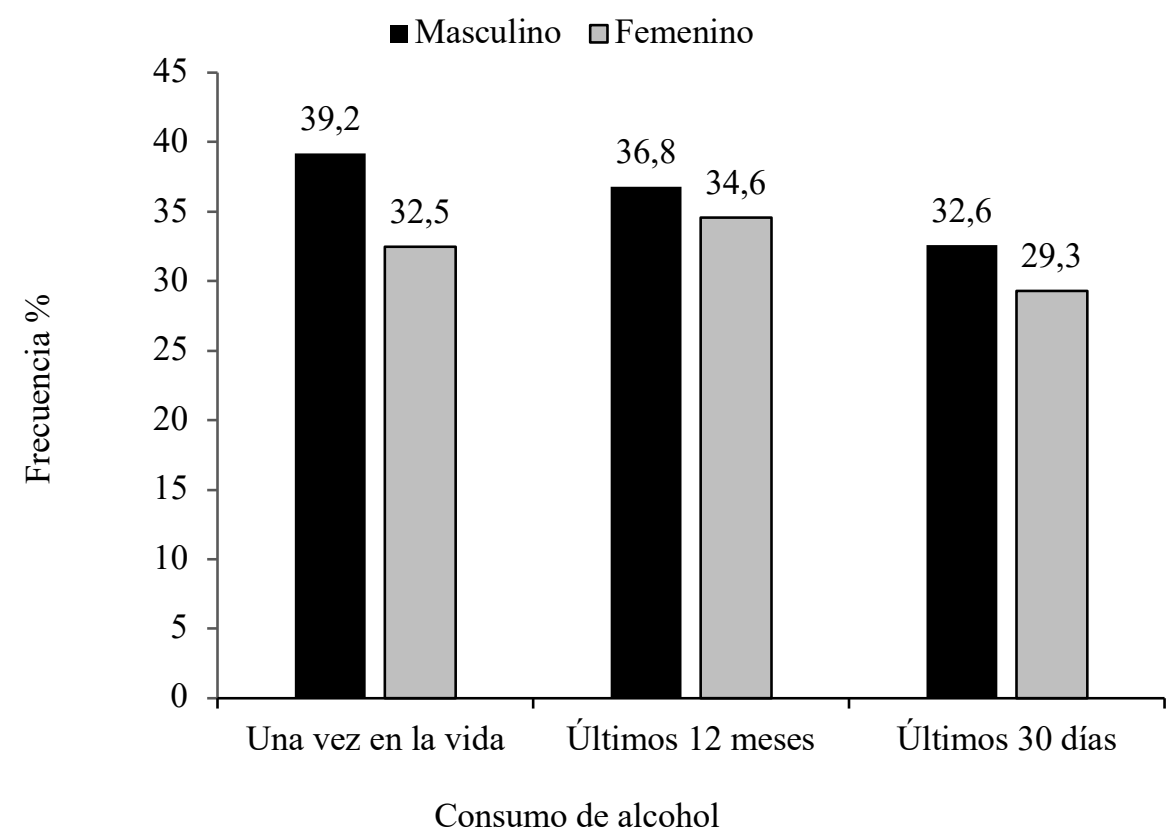

Figura 5. Prevalencia de consumo de tabaco de acuerdo con el sexo

Fuente: Elaboración propia

El inicio del consumo de alcohol mencionado por los estudiantes fue desde los seis años hasta los 16, observando la mayor frecuencia a los13, 14 y 15 años (Figura 6). 


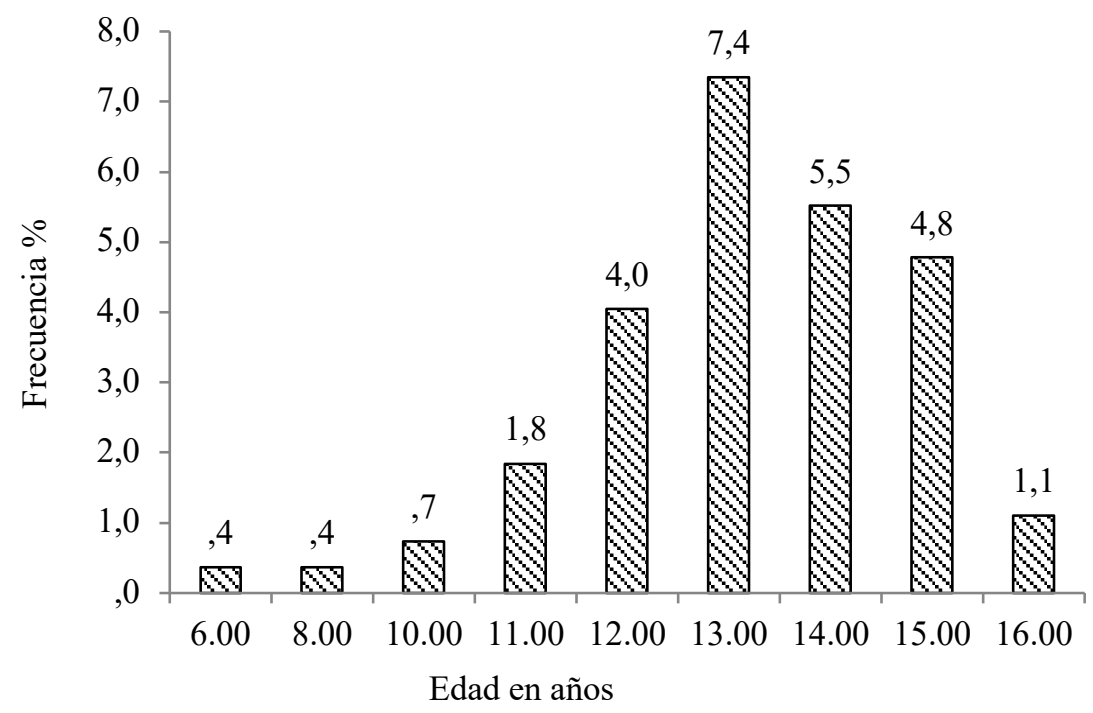

Figura 6. Inicio de consumo de bebidas alcohólicas. $\mathrm{n}=80$

Fuente: Elaboración propia

Se encontró que el consumo de marihuana entre los estudiantes fue alto a pesar de que es una droga ilícita, donde los consumidores son niños y adolescentes (Figura 7).

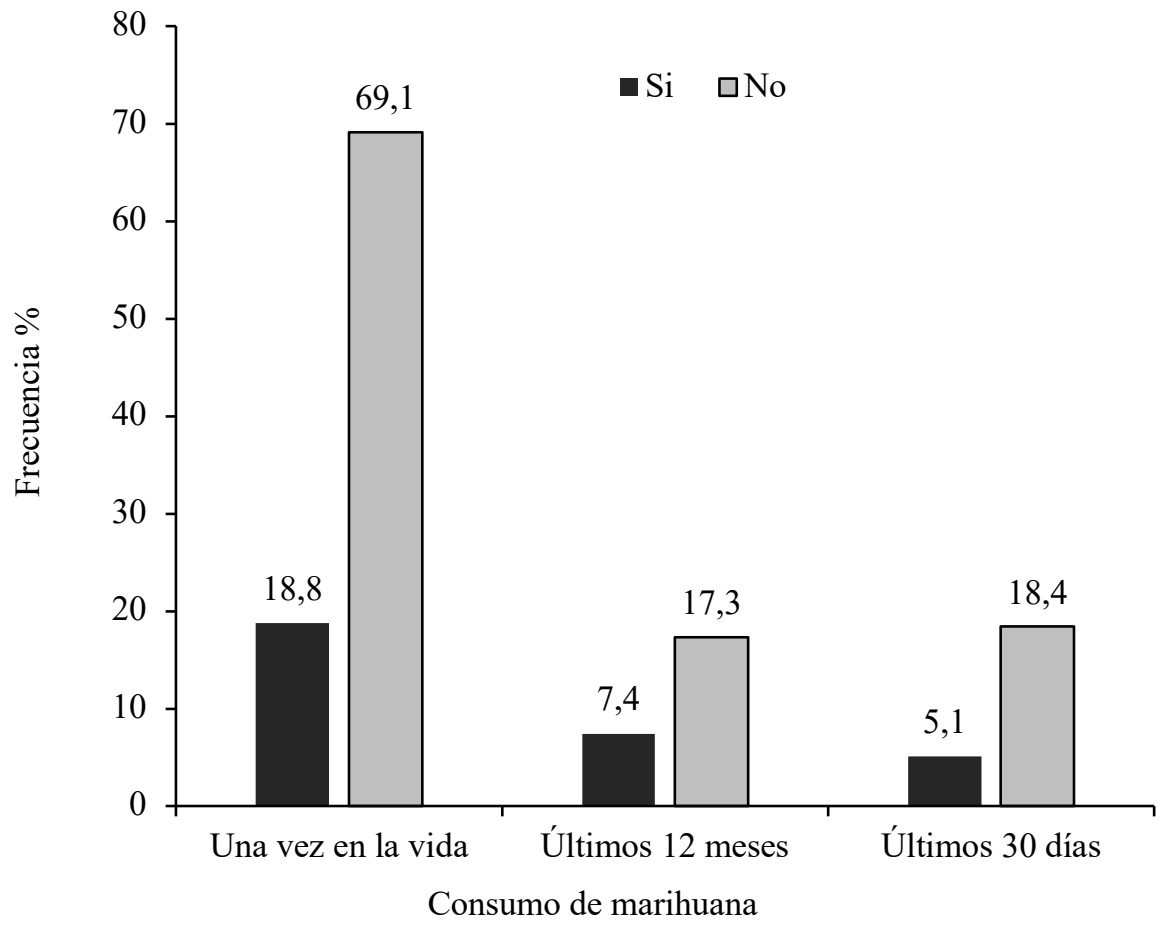

Figura 7. Prevalencia del consumo de marihuana

Fuente: Elaboración propia 
El consumo de marihuana en relación con el sexo, solamente se observaron diferencias para una vez en la vida, donde los hombres que consumieron marihuana fueron mayores al de las mujeres, pero sin diferencias estadísticas significativas. Para los últimos 12 meses y últimos 30 días, el consumo fue igual para ambos sexos (Figura 8).

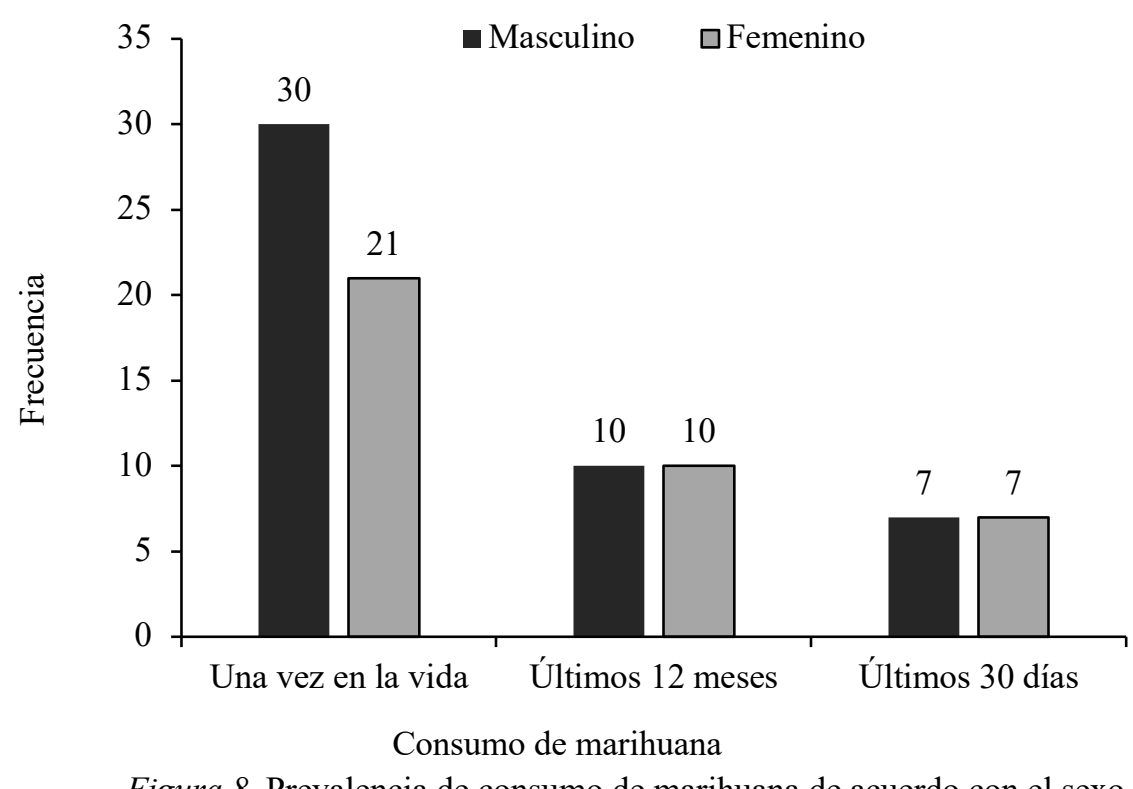

Figura 8. Prevalencia de consumo de marihuana de acuerdo con el sexo

Fuente: Elaboración propia

El inicio del consumo de marihuana fue desde los ocho años hasta los 16 años, siendo las edades de 12, 13 y 14 años donde se encuentra el mayor número de estudiantes que se iniciaron en el consumo (Figura 9).

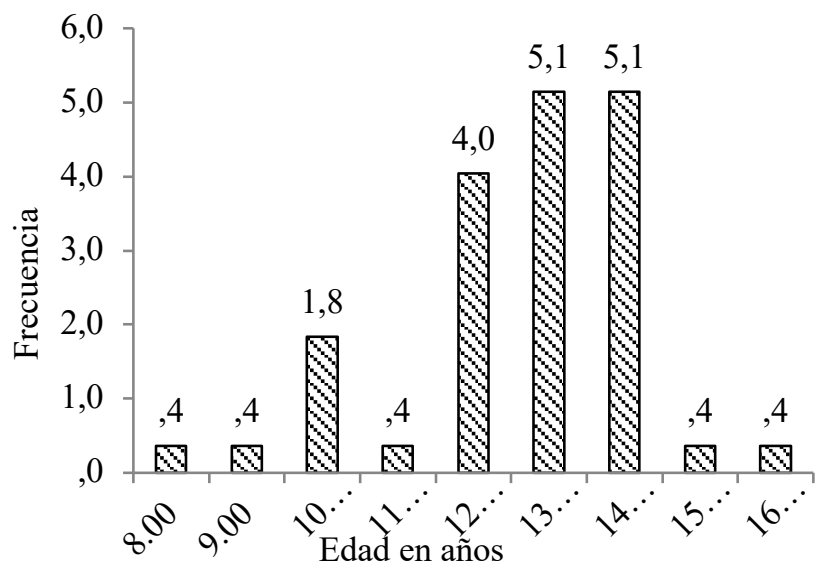

Figura 9. Inicio del consumo de marihuana. $\mathrm{n}=49$

Fuente: Elaboración propia 


\section{Discusión y conclusiones}

El inicio de consumo de alcohol, tabaco y marihuana por los estudiantes fue más frecuente a las edades de 12 a 14 años, lo que está de acuerdo con varios estudios que mencionan que el promedio de inicio del consumo de drogas en general es de 12 a 17 años (Figura 1, 4,6) por lo que recomiendan que la prevención del consumo de drogas debería iniciarse antes de los 10 años (CNA, 2010; CNA, 2001; OEA, 2004; COSA, 2000; Fundación Olof Palme, 2003). Así también otro autor menciona que las medidas preventivas deben, por tanto, dirigirse a aquellos factores específicos del inicio al consumo de estas drogas y comenzar en etapas previas a estos inicios, más específicamente la última etapa de primaria (10-12 años) y primero de secundaria (Martínez Higueras, 2012). Así también señalan que es necesario comenzar la prevención del consumo de drogas en épocas tempranas antes de que los adolescentes se puedan ver implicados de forma activa en estas conductas.

Con relación al sexo, los jóvenes varones tuvieron un consumo más alto que las féminas para la prevalencia una vez en la vida del consumo de alcohol, tabaco y marihuana (Figuras 2, 5, 8), lo cual está de acuerdo con varios autores (Alfaro Sifontes, 2009; CNA, 2010) quienes mencionan que el género masculino tiene un nivel más alto de consumo.

Para los últimos 12 meses el consumo de tabaco fue ligeramente mayor en el género femenino que el masculino (Figura 2), el consumo de alcohol fue similar en ambos géneros (Figura 5) y el consumo de marihuana fueron iguales para ambos (Figura 8).

Para los últimos 30 días el consumo de tabaco y alcohol fue similar en ambos géneros (Figura 2 y 5) y el consumo de marihuana fueron iguales (Figura 8). Esto no está de acuerdo con algunos autores que mencionan que el consumo de tabaco, alcohol y marihuana siempre ha sido mayor en el sexo masculino (Alfaro Sifontes, 2009; CNA, 2010).

Al comparar los datos obtenidos del estudio con otros realizados anteriormente (Tabla 3), se observó que el consumo para una vez en la vida, no se ha reducido a pesar de las campañas de prevención que el MINED ha realizado. En el caso de la marihuana se ha incrementado sustancialmente el consumo a nivel de los centros de estudio (CNA, 2001, 2003, 2005, 2008, 2012, 2014a y 2014b).

Tabla 3

Tendencia del consumo de tabaco, alcohol y marihuana para consumo una vez en la vida en estudios previos

\begin{tabular}{llllllll}
\hline & $2001^{*}$ & $2003^{*}$ & $2005^{*}$ & $2008^{*}$ & $2012^{*}$ & $2014^{*}$ & $2016^{* *}$ \\
\hline Tabaco & $33.1 \%$ & $36.4 \%$ & $36.40 \%$ & $27.9 \%$ & 38.0 & $31.94 \%$ & $29.0 \%$ \\
Alcohol & $29.9 \%$ & $37.4 \%$ & $45.8 \%$ & $32.5 \%$ & $61.1 \%$ & $50.94 \%$ & $32.7 \%$ \\
Marihuana & $0.1 \%$ & $4.5 \%$ & $4.5 \%$ & $5.5 \%$ & 10.00 & $10.0 \%$ & $18.8 \%$ \\
\hline
\end{tabular}

Nota: Fuente: Elaboración propia a partir de datos del CNA.

*CNA 2001, 2003, 2005,2008, 2012, 2014; **Datos obtenidos del estudio

Al relacionar el consumo de drogas en este estudio con investigaciones previas, para los últimos 12 meses durante varios años (Tabla 4) se observó que el consumo de tabaco y alcohol se ha reducido, pero en forma muy leve, sin embargo, el consumo de marihuana se ha incrementado tres veces con relación al año 2014. 
Tabla 4

Tendencia del consumo de tabaco, alcohol y marihuana para los últimos 12 meses en estudios previos

\begin{tabular}{llllllll}
\hline & $2001 *$ & $2003 *$ & $2005^{*}$ & $2008^{*}$ & $2012 *$ & $2014 *$ & $2016^{* *}$ \\
\hline Tabaco & $15.9 \%$ & $19.2 \%$ & $14.6 \%$ & $15.1 \%$ & $18.7 \%$ & $7.7 \%$ & $13.2 \%$ \\
Alcohol & $15.4 \%$ & $23.1 \%$ & $18.6 \%$ & $20.0 \%$ & $36.4 \%$ & $18.3 \%$ & $14.3 \%$ \\
Marihuana & $0.0 \%$ & $2.5 \%$ & $0.24 \%$ & $3.5 \%$ & $3.3 \%$ & $2.0 \%$ & $7.4 \%$ \\
\hline
\end{tabular}

Nota: Fuente: Elaboración propia partir de datos del CNA

*CNA 2001, 2003, 2005, 2008, 2012, 2014a; **Datos del estudio

Al cotejar el consumo encontrado en este estudio con otros previos se obtuvo para los últimos 30 días, durante varios años (Tabla 5), que el consumo ha tenido una tendencia a incrementarse en el alcohol y tabaco, pero es más evidente para la marihuana que se ha quintuplicado su consumo.

Tabla 5

Tendencia del consumo de tabaco, alcohol y marihuana, para los últimos 30 días, en estudios previos

\begin{tabular}{llllllll}
\hline & $2001^{*}$ & $2003^{*}$ & $2005^{*}$ & $2008^{*}$ & $2012^{*}$ & $2014^{*}$ & $2016^{* *}$ \\
\hline Tabaco & $9.2 \%$ & $11.7 \%$ & $11.7 \%$ & $9.3 \%$ & $12.1 \%$ & $5.0 \%$ & $5.9 \%$ \\
Alcohol & $7.1 \%$ & $16.0 \%$ & $10.1 \%$ & $11.5 \%$ & $18.6 \%$ & $9.5 \%$ & $9.6 \%$ \\
Marihuana & $0.0 \%$ & $1.1 \%$ & $0.17 \%$ & $1.8 \%$ & $0.7 \%$ & $1.3 \%$ & $5.1 \%$ \\
\hline
\end{tabular}

Nota: Fuente: Elaboración propia partir de datos del CNA

*CNA 2001, 2003, 2005,2008, 2012, 2014a; **Datos del estudio

\section{Referencias}

Alfaro Sifontes, M. (2009). Análisis de los resultados de la encuesta de prevalencia de consumo de drogas en escolares de Educación Básica y Media de Centros Educativos Urbanos 2008. Recuperado de http://www.seguridad.gob.sv/observatorio/demanda/ Estudios/INFORME\%20ANALISIS\%20RESULTADOS\%20ESCOLARES $\% 202008, \% 20 \mathrm{M}$. SIFONTE S\%202009.pdf.

Bachman, J.G., Johnston, L.D., O'Malley, P.M., y Humphrey, R.H. (1988). Explaining the recent decline in marijuana use: Differentiating the effects of perceived risk, disapproval, and general lifestyle factors. Journal of Health and Social Sciences, 29, 92-112.

Becoña Iglesias, E. (2002a). Bases teóricas que sustentan los programas de prevención de drogas. Madrid: Plan Nacional sobre Drogas. Retrieved from http://www.papelesdelpsicologo.es/ vernumero.asp?id=1424. 
Becoña Iglesias, E. (2002b). Bases científicas de la prevención de las drogodependencias. Santiago de Campostela, La Coruña: Delegación del gobierno para el Plan Nacional sobre Drogas.

Comisión Nacional Antidrogas. (2014a). Estudio nacional sobre el consumo de drogas en población en general de El Salvador-2014. Retrieved from http://www.seguridad.gob.sv/observatorio/demanda/Estudios/INFORME\%20 FINAL\%20HOGARES\%20Ebook-1.pdf.

Comisión Nacional Antidrogas. (2014b). Estudio nacional sobre consumo de drogas y de otras problemáticas en población estudiantil universitaria de El Salvador 2012. Retrieved from http:/www.seguridad.gob.sv/ observatorio/ demanda/Estudios/2014/Informe\%20Final\%20Universitarios $\% 202012 \% 20 \%$ 28ebook\%29.pdf.

Comisión Nacional Antidrogas de El Salvador, CNA (2012). Resumen Ejecutivo. Recuperado de www.cna.gob.sv.

Comisión Nacional Antidrogas. (2010). Segundo estudio nacional sobre consumo de sustancias psicoactivas en población escolar de El Salvador, SIDUC 2008. Retrieved from http:/www.seguridad.gob.sv/observatorio/ demanda/2010/Microsoft $\% 20$ Word $\% 20 \% 20$ TABLAS $\% 20$ RESULTADOS $\% 20$ ESC OLARES\%20SIDUC2008\%20_FINALDefinitivo_-1.pdf.

Comisión Nacional Antidrogas. (2008). Segundo estudio nacional sobre consumo de sustancias psicoactivas en población escolar de El Salvador, SIDUC 2008. $\begin{array}{lllll}\text { Recuperado el } 30 \quad \text { agosto } & 2014 . & \text { Retrieved from }\end{array}$ http://www.seguridad.gob.sv/observatorio/demanda/2010/Microsoft $\% 20$ Word $\% 20$ \%20TABLAS\%20RESULTADOS\%20ESCOLARES\%20SIDUC2008\%20_FINAL Definitivo_-1.pdf.

Comisión Nacional Antidrogas. (2006). Informe de avances y cumplimientos de metas en el marco del plan nacional antidrogas 2002-2008. Retrieved from http://www.seguridad.gob.sv/ observatorio/demanda/2010/Microsoft $\% 20$ Word $\% 20 \% 20$ TABLAS\%20RESULTADOS\%20ESCOLARES\%20SIDUC2008\% 20_FINALDefinitivo_-1.pdf.

Comisión Nacional Antidrogas. (2005). Sistema de datos Interamericano de datos uniformes sobre consumo de drogas en El Salvador. Retrieved from 2016, de http://www.seguridad.gob.sv/institucion/estructura-organizativa/ comision-nacionalantidrogas/.

Comisión Nacional Antidrogas. (2003). Situación del narcotráfico, prevalencias de consumo en El Salvador y tendencias en la región centroamericana. Retrieved from 2016, de http://www.seguridad.gob.sv/cna/wp-content/uploads/2018/04/ElSalvador-Situacion-Narcotrafico-y-Consumo.pdf.

Comisión Nacional Antidrogas. (2001). Datos preliminares encuesta sistema interamericano de datos uniforme sobre el consumo de drogas SIDUC Fase I. Retrieved from http://www.seguridad.gob.sv/observatorio/demanda /rdindinvestigaciones-SIDUC.htm.

Comisión Salvadoreña Antidrogas. (2000). Informe preliminar Pacardo El Salvador. Retrieved from http://www.seguridad.gob.sv/ observatorio/demanda/ inf_pacardo.htm. 
Fundación Olof Palme y Comisión Salvadoreña Antidroga (2003). Encuesta nacional de opinión de niños, niñas y adolescentes salvadoreños. Retrieved from http://www.seguridad.gob.sv/ observatorio/demanda/rdindestudios demanda.htm

Hernández, R., Fernández, C., y Baptista, P. (2014). Metodología de la investigación. $6^{a}$. Ed. México: McGraw-Hill

Martínez Higueras, I. M. (2012). Efectos de la exposición y calidad de la prevención escolar en el consumo de drogas y factores de riesgo de estudiantes de secundaria. Tesis Doctoral. Universidad Complutense de Madrid. Retrieved from http://eprints.ucm.es/15002/1/T33680.pdf.

Martínez-Lorca, M., y Alonso-Sanz, C. (2003). Búsqueda de sensaciones, autoconcepto. Asertividad y consumo de drogas ¿Existe relación? Retrieved from https://scholar.google.com/scholar_lookup?title=B\%C3\%BAsqueda $\%$

20de $\% 20$ sensaciones $\% 20$ autoconcepto $\% 20$ asertividad $\% 20 \mathrm{y} \% 20$ consumo $\% 20 \mathrm{de} \% 2$ 0drogas $\% 20 \%$ C2\%BFExiste $\% 20$ relaci $\%$ C3\%B3n?\&publication_year=2003\&hl $=$ es \&author=MART\%C3\%8DNEZLORCA\%20M\&journal=Adicciones.

Ministerio de Educación. (2008). Manual para promover y fortalecer la comunicación asertiva, entre los adolescentes y jóvenes. San Salvador: MINED.

Ministerio de Justicia y Seguridad Pública. (2009). Comisión Nacional Antidrogas. Retrieved from http://www.seguridad.gob.sv /index.php /participaciónciudadana/84.

Ministerio de Sanidad, Servicios Sociales e igualdad. (2013). Encuesta Estatal Sobre Uso de Drogas en Enseñanzas Secundarias (ESTUDES) 2012/2013, España. Retrieved from http://www.pnsd.msc.es/ Categoria2/observa/pdf/ PresentESTUDES2012_2013.pdf

Organización de Estados Americanos (2004). Informe comparativo 7 países, encuestas escolares a nivel nacional: El Salvador, Guatemala, Nicaragua, Panamá, Paraguay, República Dominicana y Uruguay 2003. Retrieved from http://www.seguridad.gob.sv/observatorio/ demanda/2004/INFORME\% 20CO MPARATIVO\%207\%20PAISES.pdf.

Organización Mundial de la Salud. (13 marzo 2017). Consumo de drogas causa medio millón me muertos anuales. Retrieved from http://www.eltiempo.com/vida/salud/muertes-por-consumo-de-drogas-segun-la-oms $\underline{-67048 .}$

Organización Mundial de la Salud. (2016). Informe mundial sobre las drogas. UNODC. Retrieved from https://www.unodc.org/doc/wdr2016/ WDR_2016_ExSum_ spanish.pdf.

Organización Mundial de la Salud. (2015). Informe mundial sobre las drogas. UNODC. Retrieved from https://www.unodc.org/documents/wdr2015/WDR15 ExSum_S.pdf.

Ortiz, N., y Silva, M. A. (2005). Significados y contradicciones del fenómeno de las drogas: drogas lícitas e ilícitas en Chile. Rev. Latino-Am. Emfermagem. 13 n. Retrieved from http://www.sumarios.org/sites/default/ files/pdfs/47909_5752.PDF.

Polit, D. F., y Hungler, B. P. (2000). Investigación científica en ciencias de la salud. ( $6^{\mathrm{a}}$. Ed.). México: McGraw-Hill Interamericana.

Quintero Ordóñez, M. B. (2015). La prevención de las adicciones en contextos familiares y escolares: Análisis del programa de prevención de la asociación Proyecto Hombre "entre todos". Tesis Doctoral. Universidad de Granada. Granada. 
República de El Salvador. (2011). Estrategia Nacional Antidrogas de El Salvador 20112015. Retrieved from www.seguridad.gob.sv/observatorio/pnad/ ENA\%2020112015.pdf.

Rivera de Parada, A. (2009). Factores de riesgo y protección del consumo de drogas entre estudiantes universitarios, salvadoreños, año 2007. Retrieved from http://dsuees.uees.edu.sv/ xmlui/handle/ 20.500.11885/ 146.

Salmerón Gómez, R. (2015). (Mini) Manual de PSPP, alternativa libre a SPSS. Retrieved from https:// estadisticasensocialesuai.files.wordpress.com/ 2016/08/pspp1.pdf.

Servicio Nacional para la Prevención y Rehabilitación del Consumo de Drogas y Alcohol. (2014). Décimo estudio nacional de drogas en población escolar. Principales resultados nacionales. Retrieved from http://www.senda.gob.cl/wpcontent/uploads/2014/07/decimo-escolares 2013.pdf.

The Survey System. (2013). Sample Size Calculator. Creative Research System. Retrieved from http://www.surveysistem.com/ sscalc.htm.

Fecha de recepción: 02/12/2019

Fecha de revisión: 04/12/2019

Fecha de aceptación: 17/01/2020 